\title{
REVISIÓN
}

\section{Una introducción a las aplicaciones industriales de los láseres. Aspectos económicos y nuevos desarrollos}

\author{
J.M. Orza ${ }^{(*)}$
}

\begin{abstract}
Resumen Se reproducen algunas cifras del mercado de láseres en 1997. Se pasa revista a treinta años de procesado de materiales metálicos con láseres de alta potencia. Se hace una referencia especial a los programas europeos de I+D y se plantean, finalmente, algunas cuestiones sobre la evolución previsible de aquí al año 2000.
\end{abstract}

Palabras clave: Mercado láser. Tratamiento láser. Tendencias futuras.

\section{An introduction to industrial applications of lasers. Economical aspects and new developments}

\begin{abstract}
The laser market forecast for 1997 is reviewed. The last thirty years have seen a continued strong growth in metallic materials processing with high power lasers. A special mention to I+D European projects is done and finally some topics about expected evolution to the 2000 year are raised up.
\end{abstract}

Keywords: Laser market forecast. Laser treatment. Future trends.

\section{INTRODUCCIÓN}

Según los datos más recientes, la cifra de ventas de láseres de todos los tipos en todo el mundo, durante 1997, se estima en unos 3,3 miles de millones de dólares (1). Al parecer, desde el punto de vista comercial los dos últimos años han sido excelentes, habiéndose superado claramente la crisis sufrida durante los primeros años del decenio de los 90. Las tablas I y II presentan las cifras, distribuidas por aplicaciones y por tipos de láseres, respectivamente. Algo más de la mitad (el $58 \%$ del mercado total) corresponde a láseres de diodos con aplicaciones en telecomunicaciones y el denominado "almacenamiento óptico" que comprende diversos productos de consumo masivo, como son el disco compacto, los sistemas de lectura y escritura de CD's, DVD's (Digital Video-Disk), etc. Más de la mitad del mercado (el $57 \%$ ) de los otros tipos de láseres, que no son de diodos, se dedica al procesado de materiales; le siguen en importancia las apli-

(*) Instituto de Estructura de la Materia, CSIC. Serrano, 123 28006-Madrid (España). caciones médicas (28\%) y las aplicaciones de I + D (investigación científica y desarrollo tecnológico), $(10 \%)$. El procesado de materiales metálicos utiliza casi exclusivamente láseres de dióxido de carbono y de estado sólido (Nd-Yag) con potencias medias del orden de kilovatios. Existen láseres comerciales de $\mathrm{CO}_{2}$ de hasta $45 \mathrm{~kW}$ (en módulos de $\sim 15 \mathrm{~kW}$ acoplables) y láseres de Nd-Yag de hasta $5 \mathrm{~kW}$. En el rango de potencias hasta $3 \mathrm{~kW}$, ambos tipos de láseres se aplican a procesos de corte, taladro y soldadura (ésta en pequeña escala). Los de más altas potencias (5 kW y superiores) son aplicables especialmente a procesos de soldadura profunda a alta velocidad. Las cifras de la tabla I que incluyen procesos como el de marcado realizables con potencias más bajas, comprenden también el procesado de materiales semiconductores y aplicaciones de microelectrónica, así como otros procesos de interés industrial que utilizan diferentes tipos de láseres: creación rápida de prototipos, manufactura desde la mesa de despacho (desktop manufacturing) y micromecanizado. Existe también un pequeño nicho para láseres de excímeros con los que se está ya iniciando la fabricación de chips de circuitos integrados de semiconductores con técnicas de foto- 
TABLA I.- Cifras estimadas del mercado de láseres y sistemas láser en 1997, clasificadas por aplicaciones, en millones de dólares. Adaptado de Laser Focus World. Ene.-Feb. 1997

TABLE I.- Worldwide commercial laser market in 1997 (from Laser Focus World, Jan.-Feb. 1997)

\begin{tabular}{|c|c|c|c|}
\hline Aplicaciones & $\begin{array}{c}\text { Láseres } \\
\text { no de } \\
\text { Diodos }\end{array}$ & $\begin{array}{c}\text { Láseres } \\
\text { de } \\
\text { Diodos }\end{array}$ & Ejemplos \\
\hline $\begin{array}{l}\text { Procesado de } \\
\text { materiales }\end{array}$ & 804,3 & 9,1 & $\begin{array}{l}\text { Corte, taladro, soldadura. Semiconductores. Microe- } \\
\text { lectrónica. }\end{array}$ \\
\hline Medicina (terapia) & 303,6 & 2,7 & Sistemas quirúrgicos, oftálmicos, de foto-radiación \\
\hline $\begin{array}{l}\text { Investigación cientí- } \\
\text { fica y técnica }\end{array}$ & 149,0 & 1,2 & Experimentación (Física, Química, Biología) \\
\hline Instrumentación & 40,8 & 4,4 & Analítica y biomédica. Clasificadores de células \\
\hline Registro de imágenes & 27,8 & 70,0 & Impresoras láser. Artes gráficas \\
\hline Sensores & 29,7 & 3,0 & $\begin{array}{l}\text { Detección remota de contaminantes. Control de emi- } \\
\text { siones. }\end{array}$ \\
\hline $\begin{array}{l}\text { Metrología, inspección } \\
\text { y control }\end{array}$ & 17,4 & 1,5 & Alineamiento. Inspección holográfica. Medida de flujos \\
\hline $\begin{array}{l}\text { Entretenimiento, } \\
\text { exhibiciones }\end{array}$ & 12,0 & 17,3 & Espectáculos Publicidad \\
\hline Telecomunicaciones & 4,0 & $1.090,0$ & Redes de comunicaciones con fibra óptica \\
\hline Almacenamiento óptico & 9,2 & 704,0 & Grabado/lectura de discos compactos (CD) \\
\hline $\begin{array}{l}\text { Lectura de código } \\
\text { de barras }\end{array}$ & 2,1 & 6,8 & Puntos de venta. Control de almacén y de procesos \\
\hline Otras & 6,5 & 11,3 & \\
\hline TOTALES & $1.406,0$ & $1.921,0$ & \\
\hline
\end{tabular}

litografía en el ultravioleta profundo. Otras aplicaciones son el recocido (annealing) de silicio a polisilicio para la producción de paneles de exhibición planos y el "microtaladro" de espitas (nozzles) para cabezales de impresoras de chorro de tinta.

\section{EL LÁSER COMO HERRAMIENTA DE PRODUCCIÓN}

Desde el punto de vista de su aplicación para el procesado de materiales, los láserés ofrecen las siguientes ventajas importantes:

a) la densidad de energía que se puede dirigir a una región localizada de un material es considerablemente mayor que la que se puede lograr por cualquier otra tecnología. La posibilidad de alcanzar muy altas temperaturas en tiempos muy breves permite el procesado con láseres de cualquier tipo de materiales a través de nuevos procedimientos de mecanizado; b) Un haz láser, en la práctica, no tiene inercia y es posible enfocarlo con precisión óptica. Consecuentemente, los láseres son ideales para su adaptación a técnicas de control automático y aplicaciones de procesado rápido de formas complejas.

Atendiendo a la cantidad de energía requerida para el proceso se pueden considerar dos categorías principales de aplicaciones: las que requieren aportes relativamente pequeños, generalmente bajo controles muy estrictos y precisos (micromecanizado e ingeniería de semiconductores, impresión y lectura ópticas y análogas), y las que transcurren con aportes energéticos sustanciales para la realización de procesos macroscópicos (como corte, soldadura, temple, recocidos, recubrimientos y aleaciones superficiales y otros). Son, estas últimas, análogas a otras operaciones convencionales en las industrias mecánicas, las que se consideran en este artículo. Las ventajas principales derivadas de la utilización de láseres se presentan en la tabla III. 
TABLA II.- Estimación del número de láseres vendidos en 1997 y de su valor, en millones de dólares. Adaptado de Laser Focus World. Ene.-Feb. 1997.

TABLE II.- Worldwide commercial laser sales and applications (from Laser Focus World, Jan.-Feb. 1997)

\begin{tabular}{|c|c|c|c|}
\hline \multicolumn{4}{|c|}{ LÁSERES (NO DE DIODOS) } \\
\hline $\begin{array}{l}\text { Tipo de láser } \\
\text { económico }\end{array}$ & Unidades & $\begin{array}{c}\text { Cifra de } \\
\text { ventas } \\
\text { (mill. de \$) }\end{array}$ & Aplicaciones principales, según su volumen \\
\hline $\begin{array}{r}\text { Dióxido de carbono: } \\
\text { sellados } \\
\text { de flujo }\end{array}$ & $\begin{array}{l}7.896 \\
3.500\end{array}$ & $\begin{array}{r}120,2 \\
3710\end{array}$ & $\begin{array}{l}\text { Proceso de materiales. Medicina. Metrología y con- } \\
\text { trol de calidad. } \\
\text { Proceso de materiales: metales y no metales }\end{array}$ \\
\hline $\begin{array}{l}\text { Estado sólido: } \\
\text { Bombeo con lámpara } \\
\text { Bombeo con láseres } \\
\text { Bombeo con diodos }\end{array}$ & $\begin{array}{r}9.569 \\
597 \\
4.753\end{array}$ & $\begin{array}{r}364,9 \\
29,8 \\
87,7\end{array}$ & $\begin{array}{l}\text { Proceso de materiales. Medicina. I+D. Sensores } \\
\text { I+D. Sensores } \\
\text { I+D. Sensores }\end{array}$ \\
\hline $\begin{aligned} \text { Iónico } & <1 \mathrm{~W} \\
& >1 \mathrm{~W}\end{aligned}$ & $\begin{array}{r}14.058 \\
2.669\end{array}$ & $\begin{array}{l}57,8 \\
65,3\end{array}$ & $\begin{array}{l}\text { Registro de imágenes. Instrumentación } \\
\text { Medicina. Entretenimiento. I+D. Artes gráficas }\end{array}$ \\
\hline $\mathrm{HeCd}$ & 3.040 & 26,4 & Impresión. Proceso de materiales. Artes gráficas \\
\hline $\mathrm{HeNe}$ & 120.100 & 30,5 & Código de barras. I+D. Instrumentación \\
\hline Vapor metálico & 105 & 0,7 & I+D. Medicina \\
\hline Colorantes & 336 & 38,1 & Medicina. I+D \\
\hline \multirow[t]{2}{*}{ Excímeros } & 1.149 & 207,5 & I+D. Medicina. Proceso de materiales \\
\hline & 167.771 & $1.406,3$ & \\
\hline LÁSERES DE DIODOS & $218 \times 10^{6}$ & 1913 & \\
\hline Longitud de onda & $\begin{array}{l}\text { Millones } \\
\text { de } \\
\text { unidades }\end{array}$ & $\begin{array}{c}\text { Cifra de } \\
\text { ventas } \\
\text { (mill. de \$) }\end{array}$ & Aplicaciones principales, según su volumen económico \\
\hline$<700 \mathrm{~nm}$ (en el visible) & 8,9 & 117 & $\begin{array}{l}\text { Entretenimiento. Lectura de códigos de barras. } \\
\text { Almacenamiento óptico. CD-ROM. DVD }\end{array}$ \\
\hline $750-950 \mathrm{~nm}<10 \mathrm{~mW}$ & 195,8 & 603 & $\begin{array}{l}\text { Almacenamiento óptico. Registro de imágenes. } \\
\text { Audio CD y CD-ROM }\end{array}$ \\
\hline$>10 \mathrm{~mW}$ & 12,8 & 96 & \\
\hline $950-1.550 \mathrm{~nm}$ & 1,2 & 1.096 & Telecomunicaciones. Instrumentación \\
\hline$>1.550$ & $3,5 \times 10^{4}$ & 0,6 & Instrumentación \\
\hline Apilamientos ("stacks") & $2,5 \times 10^{-4}$ & 0,8 & Procesado de materiales. Instrumentación \\
\hline
\end{tabular}

\section{TREINTA AÑOS DE PROCESADO DE MATERIALES CON LÁSERES}

El procesado de materiales con láseres, como realización industrial, se desarrolló inicialmente en los Estados Unidos a partir de los años setenta (2).
La correspondiente evolución en Europa y en Japón fue un tanto a remolque de aquélla. Como es bien sabido, esta nueva tecnología industrial se produce, en gran parte, en los Estados Unidos como retorno de inversiones considerables de los departamentos de Defensa y de Energía a través de contratos y 
TABLA III.- Ventajas de láser como herramienta de producción

TABLE III.-Laser advantages as a production tool

\begin{tabular}{|c|c|c|}
\hline Flexibilidad & $\begin{array}{l}\text { Rapidez de proceso y acabado } \\
\text { de calidad }\end{array}$ & $\begin{array}{c}\text { Concentración de potencia en } \\
\text { el punto preciso }\end{array}$ \\
\hline Variedad de procesos & Alta precisión (exactitud) & Alta intensidad \\
\hline Geometría arbitraria & Sin esfuerzos y al instante & Utilización dirigida \\
\hline Facilidad de automatización & Sin necesidad de retoques & Sin acciones colaterales \\
\hline $\begin{array}{l}\text { Facilidad de integración y de } \\
\text { combinación }\end{array}$ & & \\
\hline
\end{tabular}

subcontratos con empresas industriales y de la rápida transferencia de tecnología entre los sectores público y privado característica de este país.

La primera etapa de crecimiento (lento) corresponde a la utilización de láseres de estado sólido (rubí y Nd-Yag) en aplicaciones para la industria electrónica. Generalmente, en estas primeras aplicaciones era el usuario final el que desarrollaba el sistema integrando el láser en el proceso. $\mathrm{Al}$ crecer el número de aplicaciones semejantes apareció un mercado para sistemas delicados, de tipo estándar, como por ejemplo, rayado de cerámicas, ajuste de resistencias o soldaduras de cajas de relés. Sistemas de este tipo continúan ofreciéndose hoy por varios fabricantes.

Poco a poco, durante los años 70 , los láseres de $\mathrm{CO}_{2}$ comienzan a encontrar aplicaciones específicas con exigencias energéticas mayores que las suministrables por los de estado sólido; hacia el final de esta década, el número de láseres de $\mathrm{CO}_{2}$ empleados en la industria suponía, aproximadamente, el 40 $\%$ del total.

La tasa de crecimiento de ambos tipos de láseres se mantuvo en cifras superiores al $20 \%$ anual hasta 1986, en que comienzan a observarse síntomas de estancamiento en las cifras de ventas globales.

Según Belforte y asociados (3), la fabricación de láseres y sistemas láser para aplicaciones industriales empleaba en 1995 a unas 18.000 personas, el 24 $\%$ de ellas en los Estados Unidos, el $33 \%$ en Europa, el $38 \%$ en Japón y el $6 \%$ en otros países.
La tendencia a construir sistemas estandarizados se ha extendido durante los años 80 a los láseres de $\mathrm{CO}_{2}$ de diversas potencias. Existen actualmente más de 30 versiones comerciales de sistemas para corte de chapa metálica con láseres de $\mathrm{CO}_{2}$, en la gama de 1.000 a $1.500 \mathrm{~W}$, en onda continua, con sistemas de dos ejes con CNC para manejo del haz o de la chapa (con láseres de $1.500 \mathrm{~W}$ se pueden cortar chapas de hasta $12 \mathrm{~mm}$ de espesor; sistemas de óptica flotante permiten fácilmente dimensiones de $3 \times 4 \mathrm{~m}$ ). También está en auge la producción de sistemas para soldadura con láseres de $\mathrm{CO}_{2}$, con potencias de 1 a $5 \mathrm{~kW}$, con aplicación principalmente para componentes de automóviles y para conducciones y tuberías en acero inoxidable.

En la tabla IV se citan algunas de las aplicaciones más importantes. Se estima que, aproximadamente, el $60 \%$ de las aplicaciones de procesado de materiales con láseres de significación industrial se realiza con sistemas estándar, correspondiendo el resto, en su mayor parte, a sistemas láser directamente integrados en el sistema de producción o bien a sistemas únicos para nuevas aplicaciones, construidos bajo especificaciones precisas para cada tipo de aplicación. Las industrias aeronáutica, automovilística y de maquinaria pesada son las principales usuarias de este tipo de sistemas. Esta implantación del láser ha dado lugar a la creación de talleres láser (en colaboración con Universidades y centros de investigación) y que tienden a estructurarse en la forma de instalaciones

TABLA IV.- Principales firmas en el mercado de láseres industriales

TABLE IV.-Main worldwide suppliers of laser equipmens

\begin{tabular}{|llll|}
\hline \multicolumn{2}{|c|}{ Fabricantes de láseres } & \multicolumn{2}{c|}{ Fabricantes de sistemas } \\
\hline$\underline{\mathrm{CO}_{2}}$ & $\underline{\text { Nd-Yag }}$ & $\underline{\text { Corte de chapa }}$ & $\underline{\text { Marcado }}$ \\
Rofin Simar (Al) & Lumonics (R.U. y EE.UU. & Amada (Jap.) & Excel-Control Laser (EE.UU.) \\
Fanuc Oshino-Mura (Jap.) & Convergent Energy (EE.UU.) & Trumpf (Al.) & Carl Baaser Lasertechnik (Al.) \\
PRC Corp. (EE.UU.) & Rofin Sinar, Hass (Al.) & & NEC (Jap.) \\
\hline
\end{tabular}


multiláser-multipuesto con un número de estaciones de trabajo especializadas a las que se pueden dirigir, alternativa o combinadamente, los haces láser de varias fuentes que puedan tener incluso distintas características (continuas o pulsadas, potencias diferentes, etc.). Todo ello combinado con sistemas, frecuentemente automatizados, de alimentación y manejo de piezas y enlazado con sistemas de diseño y fabricación asistida por ordenador (CAD, CAM), bajo control de robots, físicamente reconocibles, es lo que permite la introducción de sistemas de manufactura flexible. Existen ya, actualmente, células y sistemas de fabricación flexible que utilizan láseres de $\mathrm{CO}_{2}$ y de Nd-Yag en procesos de producción. La aplicación más común es la de corte con láser, que suele asociarse con punzonadoras con CNC. Comienzan también a utilizarse sistemas para soldadura láser que ofrecen perspectivas de gran interés principalmente por la velocidad del proceso y la pequeña distorsión térmica que introducen. $\mathrm{Al}$ acortarse el tiempo de proceso aumentan las exigencias de la automación para manejo de materiales; si se hace con grado suficiente de flexibilidad es posible soldar en una célula dada una amplia variedad de componentes.

Como ya hemos citado, una de las principales ventajas del láser como fuente de energía y herramienta industrial es la idoneidad para su integración en sistemas automatizados. Una interesante introducción sobre este aspecto se puede ver en el texto de Steen sobre procesado de materiales con láser ${ }^{4}$. Se presentan en él, por una parte, los diversos tipos de señales indicadoras del estado del proceso y, por otra, las estrategias de control utilizables para conseguir un procesado enteramente automático, autocontrolado. Según Steen, se dispone ya de la tecnología necesaria para montar una fábrica para trabajar de forma continuada, día y noche, produciendo productos uniformes y de alta calidad, con la ayuda única de un pequeño y competente equipo (humano) de mantenimiento.

\section{ACTIVIDADES DE I+D. PROYECTOS EUROPEOS}

Un primer gran sistema experimental de fabricación flexible con láseres ha sido el montado en Japón (cofinanciado por el MITI por una cuantía de unos 55 millones de dólares en 7 años, de 1977 a 1984) con la colaboración de veinte empresas industriales bajo el liderazgo de Mitsubishi, Toshiba y Nippon Electric. Se trató con él de estudiar nuevos procedimientos de fabricación para una diversidad de productos a través de la integración de métodos convencionales y láser para mecanizado, soldadura y tratamientos térmicos. Equipado con dos láseres para procesado de materiales (uno de ellos de hasta $25 \mathrm{~kW}$ ), se desarrollaron para él diversos sistemas de control de herramientas y sistemas robóticos para inspección automática y ensamblaje.

Este proyecto japonés es un ejemplo de política nacional de promoción de la investigación en el campo de los láseres. Otras medidas de promoción comprenden desde la concesión de préstamos a bajo interés para desarrollo industrial hasta la implantación de centros nacionales de investigación con financiación estatal.

También en Europa se ha decidido acometer con subvenciones públicas programas de desarrollo tecnológico con fines civiles. El programa EUREKA, lanzado en 1985 por el presidente francés F. Mitterrand, se ha interpretado como la respuesta europea a la Iniciativa de Defensa Estratégica estadounidense. Se trata de una organización permanente que incluye en la actualidad a 26 países miembros (provenientes de la UE, la antigua EFTA y de la Europa Oriental, incluyendo a la Federación Rusa) y que se basa en las empresas industriales: son éstas las que proponen los proyectos que, si cumplen ciertas condiciones, obtendrán el "sello EUREKA". El objetivo fundamental de este programa es la promoción de la cooperación europea en proyectos de I+D próximos al mercado. Favorece la reunión de empresas comerciales, universidades, centros de investigación y fabricantes para fomentar nuevas ideas para nuevos productos. Los gobiernos y la Comisión Europea contribuyen con fondos públicos a la financiación del proyecto (dentro de cada país) de acuerdo con regulaciones aprobadas por los países participantes. Un proyecto "paraguas", aprobado desde el comienzo del programa, el EU-006 "Eurolaser", se ha expandido con el tiempo a una familia de unos 20 proyectos (dedicados principalmente al desarrollo de fuentes de alta potencia, células para procesado y regulaciones de seguridad).

Más detalles sobre los programas de ayuda, y medidas adicionales, pueden consultarse a través de Internet (http://www.cordis.lu/brite-euram).

\section{CONCLUSIONES}

En los primeros meses de 1994, se difundió entre la comunidad láser europea un interesante estudio sobre las necesidades de la industria manufacturera (de fabricación industrial) en el campo de la tecnología láser (5), apoyado con fondos del programa BRITE-EURAM. Al enjuiciar la situación actual se reconoce un avance considerable en los últimos años, aunque un tanto desigual. Junto a áreas bien establecidas (como las de corte, marcado, taladro y rayado) otras, como la soldadura y tratamientos superficiales, están aún poco desarrollados. Es necesario investigar y profundizar más, incluso en la física fundamental de estos procesos. Por otra parte, existen fallos de comunicación y de interacción entre proyectos, así como de 
coordinación entre los que son financiados con fondos públicos. Se recomienda favorecer intercambios de tecnología; proveer vías de financiación para empresas medianas y pequeñas; difundir los resultados relevantes de los proyectos subvencionados y "templar" convenientemente las prioridades en la asignación de fondos para I+D. Se ofrece también una relación de áreas y tareas en las que las diferentes organizaciones públicas de $\mathrm{I}+\mathrm{D}$ deberían asumir ciertas responsabilidades primarias, a saber:

- Fuentes (láseres de alta potencia, de $\mathrm{CO}_{2}$, de estado sólido y de excímeros; diodos y baterías de diodos, como tales y como fuentes para bombeo de láseres de estado sólido).

- Tecnología básica (nuevos métodos para remoción de calor; mejoras en la estructura de modos de los láseres de alta potencia).

- Física de la interacción láser-materia (mediciones; teoría y modelado).

- Suministro y conformación del haz láser (trayectoria del haz en sistemas de fabricación; enfoque; homogeneización e integración).

- Teoría y práctica de la soldadura autógena (metalurgia de la soldadura láser; construcción adaptada a ella; geometría del surco; soldadura guiada por sensores; aplicaciones de piezas a la medida (tailored blanks).

- Diseminación del saber (know-how) sobre aplicaciones láser (conferencias, seminarios y cursos; monografías y textos; bases de datos y sistemas de información).

- Proyectos de demostración e innovación en sistemas de manufactura con láser (láseres en CIM (computer integrated manufacturing); enfoque en la industria de metales global; enfoque en la industria automovilística).

En línea con las manifestaciones expresadas en la mencionada encuesta ha tomado cuerpo durante 1997 una iniciativa para establecer una red de comunicación de investigaciones y de experiencia en el procesado de materiales con láseres. En particular, el comité de coordinación del proyecto "paraguas" EUREKA de la Factoría Europea ha dirigido a la Comisión Europea una petición de financiación para establecer un grupo de apoyo tecnológico sobre aplicaciones láser (TSG, Technology Support Group). Un grupo diferente, el ELAN (European Laser Association Network), ha iniciado contactos con la DG XII (responsable de las actividades de I+D de la Unión) pidiendo su apoyo para actividades de cooperación y transferencia de tecnología. También existen planes de colaboración interna- cional a través del LIA (Laser Institute of America).

Es también de interés comentar brevemente una conferencia de D.A. Belforte, de noviembre de 1975: Industrial Lasers: Past, Present and Future (3). En una primera parte incide sobre la pérdida del liderazgo estadounidense frente a Europa y Japón durante los años 80 , no en la tecnología básica, pero sí en sus aplicaciones. Opina que, durante muchos años, Europa y Japón estuvieron haciendo dumping, gastando muchos millones en la investigación de aplicaciones industriales, sin que los EE.UU. emplearan en ello "ni un solo níquel"*.

Mirando al horizonte del año 2000, se cuestiona si será posible mantener hasta entonces el actual ritmo de crecimiento. Cree que algunos sectores estarán, para entonces, próximos a la saturación; por ejemplo, los talleres para corte con láser en los que ya ahora, entre el 50 y el $60 \%$ de las ventas lo son para reposición de sistemas anticuados. El siguiente gran sector de aplicaciones será el de automóviles, en el que las prospecciones sobre soldadura láser señalan un potencial de crecimiento superior al $10 \%$ anual. Buenas perspectivas también para el marcado con láseres. Pero todas estas aplicaciones no cubren más allá del $60 \%$ del mercado esperado para el año 2000. Así que habrá que introducir nuevos procesos y también nuevos sectores industriales. A la vez, esta previsión replantea la ya antigua cuestión de la velocidad de incorporación de nuevas tecnologías por parte de la industria. Sobre ello, reconoce Belforte que, en contra de sus previsiones iniciales, se tardó nada menos que 12 años en introducir (de manera generalizada) en la industria automovilística la soldadura con láseres. ¿Qué se puede hacer para reducir el tiempo necesario para una aceptación más generalizada? La respuesta es: educación del usuario final.

\section{REFERENCIAS}

(1) "Annual Survey of Laser Markets". Laser Focus World, enero y febrero 1997.

(2) E.M. Breinan, B.H. Kear y C.M. Banas. "Processing materials with lasers", Phys. Today, Nov., 44-50 (1976)

(3) D.A. Belforte et al. "Industrial Lasers: Past, Present and Future". J. Laser Applications, 7 187-191 (1995).

(4) W.M. StEen. Laser Material Processing, Cap. 7, SpringerVerlag, London 1991.

(5) LEO H.J.F. Beckman: Final report on the results of the study "Future needs of the European manufacturing industries in the field of industrial laser and laser applications". BREU-CT92-0619.

(6) "On the Growing Use of Industrial Lasers Around the World”. J. Lasers Appl. 8 (1), 6-7 (1996).

\footnotetext{
* En una intervención posterior (6) nota que la situación del mercado está cambiando y que lo hará con mayor rapidez si se resuelve satisfactoriamente el debate sobre el programa del presidente Clinton de Reinversión en Tecnología. Japón ya no se interesa como antes por la tecnología láser -sólo en el ultravioleta- y Alemania lo tiene más difícil ahora a causa del esfuerzo absorbido por la reunificación. ¿Quién dominará el mercado de mañana? Es probable que veamos el mayor desarrollo en el borde del Pacífico (Corea, Taiwan, China... El mercado se está desplazando rápidamente a las áreas con menos coste laboral).
} 